\title{
Acquisition Performance Study of Single- and Multi-carrier DS-CDMA over Nakagami- $m$ Fading Channels
}

\author{
Lie-Liang Yang and Lajos Hanzo
}

\author{
Dept. of ECS, University of Southampton, SO17 1BJ, UK. \\ Tel: +44-703-593 125, Fax: +44-703-594 508 \\ Email: 1ly@ecs.soton.ac.uk and lh@ecs.soton.ac.uk \\ http://www-mobile.ecs.soton.ac.uk
}

\begin{abstract}
In this contribution we investigate and compare the initial acquisition performance of single- and multicarrier DS-CDMA systems over multipath Nakagami$m$ fading channels under the hypothesis of multiple synchronous states in the uncertainty region of the $P N$ code. The code acquisition performance is evaluated in the context of multi-carrier DS-CDMA (MC DS-CDMA), when the correlator outputs of the subcarriers associated with the same phase of the local pseudo-noise (PN) code replica are noncoherently combined using an equal gain combining (EGC) scheme. Our investigations indicate that the code acquisition performance of the MC DS-CDMA scheme is better, than that of the single-carrier DSCDMA scheme over the multipath Nakagami- $m$ fading channels encountered. However, if the detection threshold was set inappropriately, the performance might be degraded, even if the channel quality improves.
\end{abstract}

\section{INTRODUCTION}

The acquisition problem has attracted considerable research in recent years (see [1] - [6] and the references therein). The performance of code acquisition systems has been widely investigated over Additive White Gaussian Noise (AWGN) channels and frequency non-selective, as well as frequencyselective Rayleigh or Rician fading channels, respectively. A general fading channel model, which is often used in the literature for characterizing the fading statistics in a digital mobile radio channel, is the Nakagami- $m$ distribution [7]. This model is versatile, and often better fits experimental data generated in a variety of fading environments including urban as well as indoor radio propagation channels - than the Rayleigh and Rice distributions. Moreover, the Nakagami- $m$ distribution function models a continuous transition from a Rayleigh fading channel to a Gaussian channel by varying a single parameter, namely $m$, from one to infinity [7]. Recently, the Nakagami fading channel

This work has been funded in the framework of the IST project IST-1999-12070 TRUST, which is partly funded by the European Union. The authors would like to acknowledge the contributions of their colleagues.

VTC2001, Spring, Rhodes, Greece model has been widely employed for the analysis of the error probability performance in wireless communication systems using different modulation schemes both with and without DS spreading [7][8]. In the context of initial synchronization the Nakagami- $m$ fading channel model has been invoked for the investigation of the initial synchronization performance of parallel acquisition schemes in $[4,5]$.

In this contribution we quantify the performance of a serial search acquisition scheme over multipath Nakagami- $m$ fading channels, under the hypothesis that there are multiple synchronous states $\left(H_{1}\right.$ cells) in the uncertainty region of the PN code [3]. The acquisition performance is evaluated and compared for both single-carrier DS-CDMA systems and high-data rate MC DS-CDMA systems. Studying the acquisition performance of MC DS-CDMA is expected to attract further attention, since MC DS-CDMA transmission schemes have been proposed, in order to achieve further advantages in terms of bandwidth efficiency, frequency diversity, reduced complexity parallel signal processing and interference rejection capability in high data-rate transmissions $[8,9]$.

\section{SYSTEM DESCRIPTION}

\subsection{Transmitted Signal}

The transmitter schematic of the $k$ th user is shown in Fig.1 for the MC DS-CDMA system considered, which can be viewed as the simplified transmitter schematic of the multitone and orthogonal MC DS-CDMA schemes [9]. At the transmitter side, the binary data stream having a bit duration of $T_{b}$ is serial-to-parallel converted to $U$ parallel substreams. The new bit duration of each sub-stream or the symbol duration is $T_{s}=U T_{b}$. Note that if $U=1$, then the transmitter does not include serial-to-parallel conversion and the corresponding MC DS-CDMA scheme, consequently, represents the conventional single-carrier DS-CDMA scheme. After serial-to-parallel conversion, the $u$ th substream modulates a subcarrier frequency $f_{u}$ using Binary Phase Shift Keying (BPSK) for $u=1,2, \ldots, U$. Then, the $U$ subcarrier-modulated sub-streams are multiplexed in the frequency domain, in order to form the complex modulated signal. Finally, spectral spreading is imposed on the complex signal by multiplying it with a spreading code. There- 


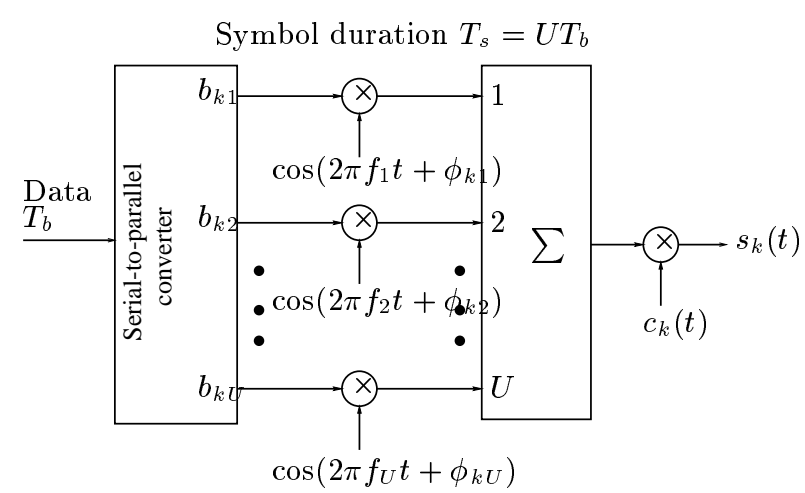

Figure 1: The $k$ th user's transmitter schematic for the generalized MC DS-CDMA system.

fore, the transmitted signal of user $k$ can be expressed as

$$
s_{k}(t)=\sum_{u=1}^{U} \sqrt{2 P} b_{k u}(t) c_{k}(t) \cos \left(2 \pi f_{u} t+\phi_{k u}\right),
$$

where $P$ represents the transmitted power per subcarrier, while $\left\{b_{k u}(t)\right\}, c_{k}(t),\left\{f_{u}\right\}$ and $\left\{\phi_{k u}\right\}$ represent the data stream, the DS spreading waveform, the subcarrier frequency set and the phase angles introduced in the carrier modulation process. The data stream's waveform $b_{k u}(t)=$ $\sum_{i=-\infty}^{\infty} b_{k u} P_{T_{s}}\left(t-i T_{s}\right)$ consists of a sequence of mutually independent rectangular pulses of duration $T_{s}$ and of equiprobable amplitudes of +1 or -1 . The spreading sequence $c_{k}(t)=\sum_{j=-\infty}^{\infty} c_{k j} P_{T_{c}}\left(t-j T_{c}\right)$ denotes the signature sequence waveform of the $k$ th user, where $c_{k j}$ assumes equiprobable values of +1 or -1 , while $P_{T_{c}}(t)$ is the rectangular chip waveform, which is defined over the interval $\left[0, T_{c}\right)$.

Let $N=T_{s} / T_{c}$ be the spreading gain of the subcarrier signals in the generalized MC DS-CDMA system and $N_{1}=$ $T_{b} / T_{c 1}$ be the spreading gain of a corresponding singlecarrier DS-CDMA system, where $T_{c 1}$ represents the chipduration of the corresponding single-carrier DS-CDMA signal. In the following analysis - for the sake of simplicity we assume that there exists no spectral overlap between the spectral main-lobes of two adjacent subcarriers in the MC DS-CDMA system. Moreover, we assume that each subcarrier signal occupies an equal bandwidth and the total system bandwidth is evenly divided amongst the $U$ number of subcarriers. Hence, we have $2 / T_{c 1}=U \times 2 / T_{c}$ or $T_{c}=U T_{c 1}$ and $N_{1}=N$, since $T_{s}=U T_{b}$. Based on the above assumption, we can infer that both the MC and the corresponding single-carrier DS-CDMA system have the same information rate of $1 / T_{b}$ and the same system bandwidth of $2 / T_{c 1}$, which allows their direct comparison in our forthcoming discourse.

\subsection{Channel Model}

We assume that the channel between the $k$ th transmitter and the corresponding receiver is a multipath Nakagami- $m$ fading channel [7]. The complex low-pass equivalent representation of the channel impulse response (CIR) experienced by subcarrier $u$ of user $k$ is given by [10]

$$
h_{k u}(t)=\sum_{l_{p}=0}^{L_{p}-1} \alpha_{u l_{p}}^{(k)} \delta\left(t-\tau_{k l_{p}}\right) \exp \left(-j \psi_{u l_{p}}^{(k)}\right),
$$

where $\alpha_{u l_{p}}^{(k)}, \tau_{k l_{p}}$ and $\psi_{u l_{p}}^{(k)}$ represent the attenuation factor, delay and phase-shift for the $l_{p}$ th multipath component of the channel, respectively, while $L_{p}$ is the total number of diversity paths and $\delta(t)$ is the Kronecker Delta-function. Let $T_{m}$ be the maximum delay-spread of the communication channel. Then, the number of resolvable paths, $L_{p}$, associated with the MC DS-CDMA signal is given by $L_{p}=$ $\left\lfloor T_{m} / T_{c}\right\rfloor+1[4,5]$, where $\lfloor x\rfloor$ represents the largest integer not exceeding $x$. The number of resolvable paths, $L_{1}$, in the context of the corresponding single-carrier DS-CDMA signal is given by $L_{1}=\left\lfloor T_{m} / T_{c 1}\right\rfloor+1=\left\lfloor U T_{m} / T_{c}\right\rfloor+1$.

We assume that the phases $\left\{\psi_{u l_{p}}^{(k)}\right\}$ in (2) are independent identically distributed (i.i.d) random variables uniformly distributed in the interval $[0,2 \pi)$, while the $L_{p}$ multipath attenuations $\left\{\alpha_{u l_{p}}^{(k)}\right\}$ in (2) are independent Nakagami random variables with a Probability Density Function $(\mathrm{PDF})$ of $[7][8]$

$$
\begin{aligned}
p\left(\alpha_{u l_{p}}^{(k)}\right) & =M\left(\alpha_{u l_{p}}^{(k)}, m, \Omega_{u l_{p}}^{(k)}\right), \\
M(R, m, \Omega) & =\left(\frac{m}{\Omega}\right)^{m} \frac{2 R^{2 m-1}}{\Gamma(m)} \exp \left(-\frac{m R^{2}}{\Omega}\right),
\end{aligned}
$$

where $\Gamma(\cdot)$ is the gamma function [10] and $m$ is the Nakagami$m$ fading parameter that characterizes the severity of the fading over the $l_{p}$-th resolvable path [7]. The parameter $\Omega_{u l_{p}}^{(k)}$ in (3) is the second moment of $\alpha_{u l_{p}}^{(k)}$, i.e., $\Omega_{u l_{p}}^{(k)}=$ $E\left[\left(\alpha_{u l_{p}}^{(k)}\right)^{2}\right]$. We assume a negative exponentially decaying multipath intensity profile (MIP) distribution given by $\Omega_{u l_{p}}^{(k)}=\Omega_{0} e^{-\eta l_{p}}, \quad \eta \geq 0$, where $\Omega_{0}$ is the average signal strength corresponding to the first resolvable path and $\eta$ is the rate of average power decay.

We assume that the system supports $K$ users and that all users have the same number of subcarriers, namely $U$. Moreover, we assume that the first user is the one that requires initial synchronization, while the other users are synchronized and reached the data transmission stage. Furthermore, we assume that all users have the same average received power. Consequently, when $K$ signals obeying the form of (1) are transmitted over the frequency-selective fading channels characterized by (2), the received signal at the base station can be expressed as

$$
\begin{array}{r}
r(t)=\sum_{k=1}^{K} \sum_{u=1}^{U} \sum_{l_{p}=0}^{L_{p}-1} \sqrt{2 P} \alpha_{u l_{p}}^{(k)} b_{k u}\left(t-\tau_{k l_{p}}\right) c_{k}\left(t-\tau_{k l_{p}}\right) \\
\cdot \cos \left(2 \pi f_{u} t+\varphi_{u l_{p}}^{(k)}\right)+n(t),
\end{array}
$$

where $\varphi_{u l_{p}}^{(k)}=\phi_{k u}-\psi_{u l_{p}}^{(k)}-2 \pi f_{u} \tau_{k l_{p}}$, which is assumed to be an i.i.d random variable having a uniform distribution in $[0,2 \pi)$, while $n(t)$ represents the AWGN with zero mean and double-sided power spectral density of $N_{0} / 2$.

\section{SERIAL SEARCH ACQUISITION}

\subsection{Search and Detection Modes}

The block diagram of the serial search mode and the detection mode for our MC DS-CDMA system is shown in Fig. 2. The received signal is first down-converted into in-phase (I) 


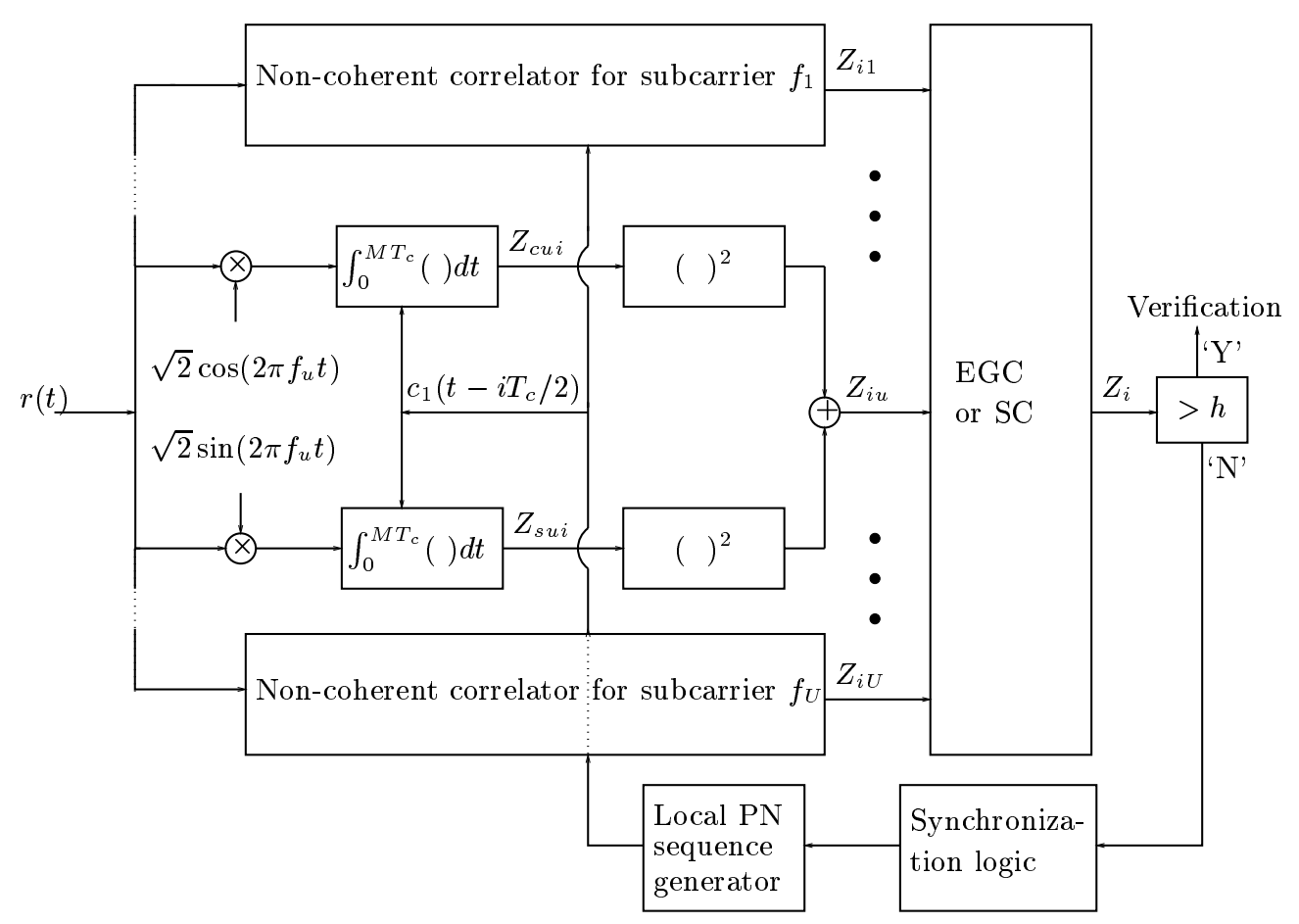

Figure 2: Block diagram of generating the decision variable $Z_{i}$ for the MC DS-CDMA code-acquisition system.

and quadrature $(\mathrm{Q})$ components associated with each subcarrier. Let us assume that the search step size is $T_{c} / 2$. For each subcarrier a pair of I-Q correlators perform correlation between the locally generated PN sequence $c\left(t-i T_{c} / 2\right)$ and the I as well as the Q baseband signals in Fig. 2, where integration takes place over the interval of $\tau_{D}=M T_{c}$ seconds, and where $\tau_{D}$ represents the integral dwell time, while $M$ is an integer. The outputs of the I-Q correlators are then squared and summed to give an output variable, which is denoted by $Z_{i u}, u=1,2, \ldots, U$ in Fig. 2 .

We assume that the transmitter aids the initial synchronization by transmitting the phase-coded carrier signal without data modulation at the beginning of each transmission. In the MC DS-CDMA acquisition scheme of Fig. 2, since all subcarriers employ the same spreading code, as shown in Fig.1, and since we have assumed that no data modulation is imposed during the acquisition stage, the outputs of the $U$ non-coherent subcarrier correlators seen in Fig. 2, which are associated with the same phase of the local despreading code can be combined. Again, in this contribution, non-coherent EGC schemes are investigated. Let $Z_{i}, i=1,2, \ldots$ be the output of the combiner, which represents the decision variable. Furthermore, let $h$ in Fig. 2 be a decision threshold. Then, the search and detection mode can be described as follows. Whenever the decision variable $Z_{i}$ exceeds the threshold $h$, the system assumes that the corresponding delay of the locally generated PN sequence is the correct delay and proceeds to the verification mode. Otherwise, if $Z_{i}$ does not exceed $h$, the relative phase of the locally generated $\mathrm{PN}$ sequence is readjusted, in order to update the decision variable $Z_{i}$, and the above process is repeated.

\subsection{Mean-Acquisition Time}

The generalized asymptotic equation for the mean acquisition time of serial search acquisition schemes has been given in [2] and [3]. It can be shown that, under the hypothesis of multiple $H_{1}$ cells in the uncertainty region of the PN sequence, the mean acquisition time can be asymptotically expressed as

$$
\bar{T}_{a c q} \approx \frac{\left[1+P_{M}(\lambda)\right]\left(1+J P_{F A}\right)}{2\left[1-P_{M}(\lambda)\right]}\left(q \tau_{D}\right)
$$

where $q$ represents the total number of states in the uncertainty region of the PN sequence, while $P_{F A}$ represents the false alarm probability of an asynchronous state $\left(H_{0}\right.$ cell). More explicitly, the false alarm probability characterizes the probability of the event, when albeit a high correlation was registered, this falsely indicated the correct synchronization instant due to channel impairments. Furthermore, $\tau_{D}=M T_{c}$ represents the integral dwell-time, while $J \tau_{D}$ represents the 'penalty time' associated with noticing that there is a false alarm and with re-entering the search mode. Moreover, $P_{M}($ ) represents the overall miss probability of the search mode, i.e. that of the event, when none of the correctly synchronized phases have been found during the tests over the whole uncertainty region. Let us assume that there are $\lambda H_{1}$ cells and $(q-\lambda) H_{0}$ cells in the total of $q$ number of states. Let the detection probabilities corresponding to the $\lambda H_{1}$ cells be expressed as $\left\{P_{D_{1}}, P_{D_{2}}, \ldots, P_{D_{\lambda}}\right\}$. Assuming that the test of each cell is an independent event, the overall miss probability is defined as

$$
P_{M}(\lambda)=\prod_{i=1}^{\lambda}\left(1-P_{D_{i}}\right) .
$$


For our MC DS-CDMA system using $U$ number of subcarriers and EGC the $U$ branches associated with the same phase of the local PN sequence are equally weighted and then added, in order to form the decision variables $Z_{i}$, as shown in Fig.2, which can be expressed as:

$$
Z_{i}=\sum_{u=1}^{U} Z_{i u}, i=1,2, \ldots
$$

The detection probability of the $H_{1}$ cell associated with the $l_{p}$ th resolvable path can be expressed as

$$
\begin{aligned}
& P_{D_{l_{p}}}=\frac{\exp \left(-\frac{m h}{2 m+\bar{\gamma}_{c} e^{-\eta l_{p}}}\right)}{\left(1+\bar{\gamma}_{c} e^{-\eta l_{p}} / 2 m\right)^{U(m-1)}} \sum_{n=0}^{\infty} \sum_{k=0}^{U+n-1} \\
& \cdot\left(-\frac{\bar{\gamma}_{c} e^{-\eta l_{p}}}{2 m}\right)^{n}\left(\frac{m}{2 m+\bar{\gamma}_{c} e^{-\eta l_{p}}}\right)^{k} \frac{(U-U m)_{n} h^{k}}{n ! k !}
\end{aligned}
$$

where $\bar{\gamma}_{c}$ represents the average SNR per chip and is given by

$$
\bar{\gamma}_{c}=\frac{9}{16}\left(\frac{1}{3 M}\left[K q\left(L_{p}, \eta\right)-1\right]+\frac{1}{2 M \Omega_{0} \gamma_{c}}\right)^{-1}
$$

where $q\left(L_{p}, \eta\right)=\frac{1-e^{-\eta L_{p}}}{1-e^{-\eta}}[8]$.

Since each resolvable path contributes two $H_{1}$ cells [2, $3]$, and since the detection probability in the context of these two $H_{1}$ cells is the same, consequently, according to (6) the overall miss probability corresponding to the $L_{p}$ number of resolvable paths can be expressed as

$$
P_{M}\left(2 L_{p}\right)=\prod_{l_{p}=0}^{L_{p}-1}\left(1-P_{D_{l_{p}}}\right)^{2}
$$

where $P_{D_{l_{p}}}$ is given by (8).

The false alarm probability of the multicarrier DS-CDMA system using EGC can be expressed as

$$
P_{F A}=\exp \left(-\frac{h}{2}\right) \sum_{k=0}^{U-1} \frac{(h / 2)^{k}}{k !} .
$$

Finally, the mean acquisition time of the serial search mode for the MC DS-CDMA using EGC can be evaluated by substituting (10) and (11) and the other related parameters into (5).

\section{NUMERICAL RESULTS}

Fig. 3 shows the influence of the fading parameter, $m$, and that of the number of subcarriers, $U$, on the overall miss probability for the EGC scheme, where $m=1,2$ and 50 represent the scenarios of Rayleigh fading, Rician fading and near-AWGN channels, respectively. From the results we observe that for the EGC scheme assuming a sufficiently high SNR/chip value, the overall miss probability decreases, when $m$ or $U$ increases. We can obtain at least $2 \mathrm{~dB} \mathrm{SNR} /$ chip gain over the whole $\mathrm{SNR} /$ chip range considered, when $U$ is increased from one to two, or from two to four, respectively.

In Fig. 4 we evaluated and compared the overall miss probability versus the normalized threshold performance of both the single-carrier and MC DS-CDMA systems for

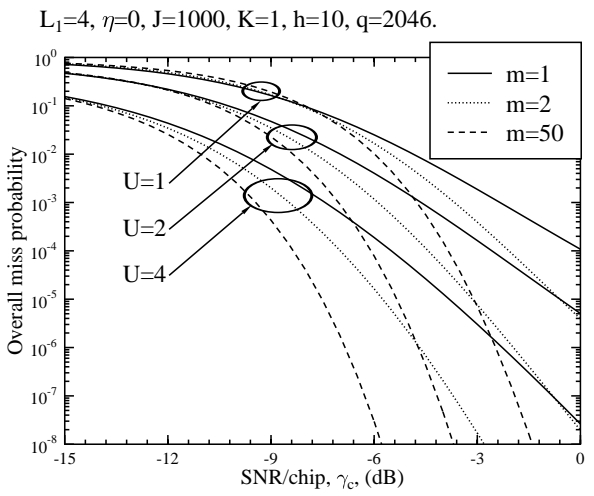

Figure 3: Overall miss probability versus the SNR per chip, $\gamma_{c}$, performance for the single-carrier serial search acquisition $(U=1)$ and for the $\mathrm{MC}(U=2,4)$ DS-CDMA schemes over multipath Nakagami- $m$ fading channels using $m=1,2,50$.

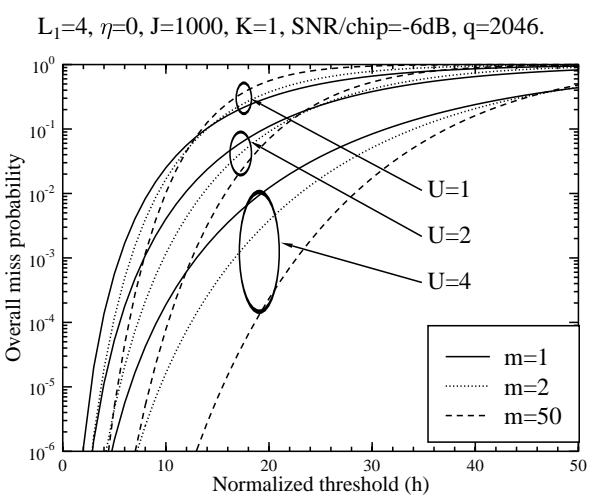

Figure 4: Overall miss probability versus the normalized threshold, $h$, performance for the single-carrier serial search acquisition $(U=1)$ and for the $\mathrm{MC}(U=2,4)$ DS-CDMA schemes over multipath Nakagami- $m$ fading channels using $m=1,2,50$.

the Rayleigh fading, Rician fading and near-AWGN channel scenarios. As expected, the overall miss probability of EGC increases for a given value of $U$ and for a given value of $m$, as the threshold, $h$, increases. In addition to our observations in Fig.3 with respect to $U$ and $m$, in Fig.4 we observe a crossover-point for the curves associated with $m=1,2$ and 50 for any given value of $U$. Observe that for the threshold values to the left of the crossover-point, the overall miss probability decreases, when the channel conditions become better, i.e. upon increasing the value of $m$. By contrast, for threshold values to the right of the crossover-point, the overall miss probability increases, when the channel conditions improve. From the results we notice furthermore that the crossover-point moves to the right, when increasing the number of subcarriers combined.

Figure 5 shows the mean acquisition time performance versus the normalized threshold $h$ and the SNR/chip for the 

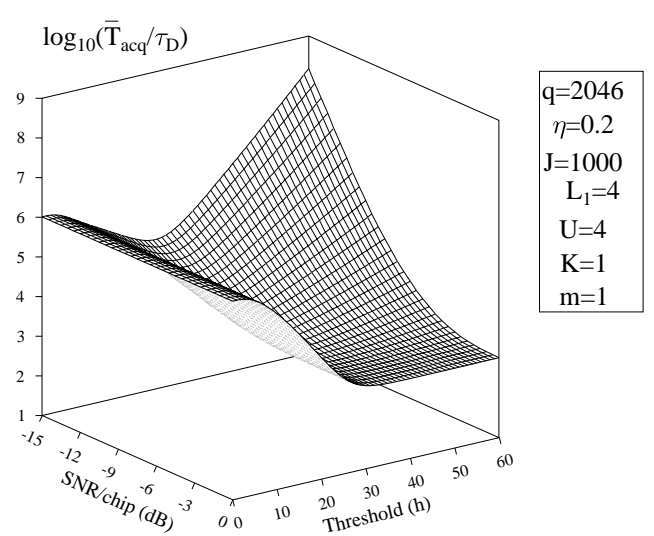

Figure 5: Mean acquisition time versus the SNR per chip, $\gamma_{c}$, and versus the normalized threshold, $h$, performance for the serial search acquisition of MC $(U=4)$ DS-CDMA over multipath Rayleigh $(m=1)$ fading channels.

MC DS-CDMA system using $U=4$ subcarriers. It is clear from Fig. 5 that an inappropriate choice of the detection threshold $h$ can lead to severe increase of the mean acquisition time, but the sensitivity of the mean acquisition time to the threshold decreases, as the SNR/chip increases. For any given SNR/chip value there exists an optimal choice of the threshold $h$, which minimizes the mean acquisition time. In addition, for any given threshold $h$, the mean acquisition time decreases, as the SNR/chip increases, and finally can reach a residual value, which is essentially due to the 'penalty time' associated with switching back to the search mode after a false alarm. This value can be computed from (5) by setting $P_{M}(\lambda)=0$, resulting in $\bar{T}_{a c q} \approx \frac{(1+J \alpha)}{2} \cdot\left(q \tau_{D}\right)$.

Figure 6 presents the mean acquisition time performance of the single-carrier and MC DS-CDMA schemes against the normalized threshold $h$. For any given number of subcarriers combined, and for a given value of $m$, there is an optimal choice of the threshold $h$, which leads to the minimum mean acquisition time. At the optimal value of $h$ we notice that for the single-carrier DS-CDMA scheme $(U=1)$ the mean acquisition time performance is degraded upon increasing the value of $m$, while for the MC DS-CDMA scheme using $U=2,4$ and the optimum threshold, the mean acquisition time performance is slightly improved, when increasing the value of $m$. Furthermore, if the value of the threshold is set inappropriately, the mean acquisition time will significantly increase, which is further aggravated upon increasing the value of $m$ for $h$-values in excess of the optimum. However, as we noticed in the context of Fig.6, the mean acquisition time performance of the EGC scheme is improved significantly and becomes more robust to the threshold for values in excess of the optimum, when more subcarrier signals are combined.

\section{REFERENCES}

[1] D. V. Sarwate, "Acquisition of direct-sequence spreadspectrum signals," in Wireless Communication TDMA versus CDMA (S. G. Glisic and P. L. Leppa-

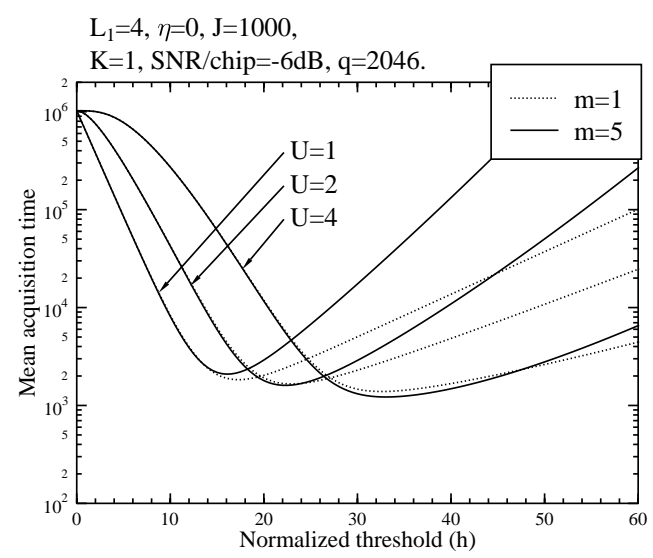

Figure 6: Mean acquisition time versus the normalized threshold, $h$, performance for the single-carrier serial search acquisition $(U=1)$ and for the MC $(U=2,4)$ DS-CDMA schemes over multipath Nakagami- $m$ fading channels using $m=1,5$.

nen, eds.), pp. 121-145, Kluwer Academic Publishers, 1997.

[2] L.-L. Yang and L. Hanzo, "Serial acquisition of DSCDMA signal in multipath fading mobile channels," To appear in IEEE Trans. on Vehi. Tech., (http://wwwmobile.ecs.soton.ac.uk/lly/).

[3] L.-L. Yang and L. Hanzo, "Serial acquisition techniques for DS-CDMA signals in frequency-selective multi-user mobile channels," in Proc. of IEEE VTC'g9, (Houston, USA), pp. 2398-2402, May 1999.

[4] L.-L. Yang and L. Hanzo, "Performance of parallel code acquisition schemes for multicarrier CDMA over frequency-selective Rayleigh fading channels", Proc. of PIMRC'2000, (London, UK), pp. 119-123, Sept. 2000.

[5] L.-L. Yang and L. Hanzo, "Parallel code-acquisition for multicarrier DS-CDMA systems communicating over multipath Nakagami fading channels," Proc. of IEEE GLOBECOM'2000, San Francisco, California, USA, Nov. 27 - Dec. 1, 2000, pp 890-894

[6] D. Lee and L. B. Milstein, "Analysis of a multicarrier DS-CDMA code-acquisition system," IEEE Trans. on Commun., vol. 47, pp. 1233-1244, August 1999.

[7] M. K. Simon and M.-S. Alouini, "A unified approach to the probability of error for noncoherent and differentially coherent modulation over generalized fading channels," IEEE Trans. on Commun., vol. 46, pp. 1625-1638, December 1998.

[8] L.-L. Yang and L. Hanzo, "Blind joint soft-detection assisted slow frequency-hopping multicarrier DSCDMA," IEEE Trans. on Commun., Vol. 48, No. 9, pp.1520-1529, Sept. 2000.

[9] R. Prasad and S. Hara, "Overview of multicarrier CDMA," IEEE Commun. Mag., pp. 126-133, December 1997.

[10] J. G. Proakis, Digital Communications. McGraw Hill, 3rd ed., 1995. 